Bull. Korean Math. Soc. 52 (2015), No. 4, pp. 1241-1252

http://dx.doi.org/10.4134/BKMS.2015.52.4.1241

\title{
DYNAMICAL BIFURCATION OF THE ONE DIMENSIONAL MODIFIED SWIFT-HOHENBERG EQUATION
}

\author{
YunCheRl CHOI
}

\begin{abstract}
In this paper, we study the dynamical bifurcation of the modified Swift-Hohenberg equation on a periodic interval as the system control parameter crosses through a critical number. This critical number depends on the period. We show that there happens the pitchfork bifurcation under the spatially even periodic condition. We also prove that in the general periodic condition the equation bifurcates to an attractor which is homeomorphic to a circle and consists of steady states solutions.
\end{abstract}

\section{Introduction}

The formation of patterns in non-equilibrium systems is closely related to the instability ([9]) and the bifurcation analysis plays an important role in understanding the instability. Indeed, the instability arises when stable states are driven into unstable states during phases transition. As a control parameter related to the instability passes through critical values, the trivial state loses its stability and bifurcates to nontrivial states which form patterns. Then the dynamics of the system after the threshold of bifurcation is completely determined by its behavior on the center manifold. In particular, Ma and Wang showed in [13] that the system bifurcates to a nontrivial attractor on the center manifold which determines the final patterns of the system.

The Swift-Hohenberg equation is a widely accepted model in the study of the formation of patterns $[1,12]$. It was derived in [18] as an approximate model for the Rayleigh-Bénard convection describing the pattern formation in layer fluids between horizontal plates. It has attracted a lot of interest in various areas of application regarding pattern formations such as Taylor-Couette flow and lasers [5]. In particular, there has been much efforts on the bifurcation analysis as a way of understanding pattern formations. See $[4,8,10,11,14,15,19,20]$ for recent development in this direction.

Received August 26, 2014.

2010 Mathematics Subject Classification. Primary 37G35, 35B32.

Key words and phrases. modified Swift-Hohenberg equation, dynamic bifurcation, center manifold function. 
In this paper, as a variation of the Swift-Hohenberg equation, we are interested in a one dimensional modified Swift-Hohenberg equation (MSHE):

$$
u_{t}=\alpha u-\left(1+\partial_{x x}\right)^{2} u+\mu u_{x}^{2}-u^{3} .
$$

Here, $u: \mathbb{R} \times[0, \infty) \rightarrow \mathbb{R}, \alpha \in \mathbb{R}$ is a control parameter related to the driving force of the system, and $\mu \in \mathbb{R}$, reminiscent of the Kuramoto-Sivashinsky equation, is a constant causing stable hexagonal patterns. The MSHE arises in the study of various pattern formation phenomena involving some kind of phase turbulence or phase transition ([6]). If $\mu=0$, then (1.1) corresponds to the usual Swift-Hohenberg equation.

In this paper, we consider the MSHE (1.1) under the periodic boundary condition on $\Omega=[-\lambda, \lambda]$, i.e., $u(-\lambda, t)=u(\lambda, t)$ for all $t \geq 0$ and some $\lambda>0$. For the functional setting of periodic MSHE, let

$$
\begin{aligned}
H & =\left\{u \in L^{2}(\Omega ; \mathbb{R}): u(-\lambda)=u(\lambda)\right\}, \\
H_{\text {per }}^{4}(\Omega ; \mathbb{R}) & =\left\{u \in H^{4}(\Omega ; \mathbb{R}): \frac{\partial^{j} u}{\partial x^{j}}(-\lambda)=\frac{\partial^{j} u}{\partial x^{j}}(\lambda) \text { for } j=0,1,2,3\right\}, \\
H_{1} & =H_{\text {per }}^{4}(\Omega ; \mathbb{R}) \cap H .
\end{aligned}
$$

On the other hand, it is easy to see that the MSHE (1.1) is invariant under the even periodic condition. So we define

$$
\begin{aligned}
\tilde{H} & =H \cap\left\{u \in L^{2}(\Omega ; \mathbb{R}): u(-x)=u(x), x \in[0, \lambda]\right\}, \\
\tilde{H}_{1} & =\tilde{H} \cap H_{1} .
\end{aligned}
$$

We formulate (1.1) in an abstract equation

$$
\left\{\begin{aligned}
\frac{d u}{d t} & =\mathcal{L}_{\alpha} u+G(u), \\
u(0) & =u_{0},
\end{aligned}\right.
$$

by setting $\mathcal{L}_{\alpha} u=-A u+B_{\alpha} u$, and

$$
\begin{aligned}
A & =\left(\frac{\partial^{2}}{\partial x^{2}}+I\right)^{2}: H_{1} \rightarrow H\left(\tilde{H}_{1} \rightarrow \tilde{H}, \text { resp. }\right), \\
B_{\alpha} & =\alpha I: H_{1} \rightarrow H\left(\tilde{H}_{1} \rightarrow \tilde{H}, \text { resp. }\right) .
\end{aligned}
$$

We also define the nonlinear operator $G(u)=G_{2}(u, u)+G_{3}(u, u, u)$, where

$$
G_{2}(u, v)=\mu u_{x} v_{x} \text {, and } G_{3}(u, v, w)=-u v w .
$$

It is easy to check that $A, B_{\alpha}, G: H_{1} \rightarrow H\left(\tilde{H}_{1} \rightarrow \tilde{H}\right.$, resp. $)$ are well defined. The global well-posedness was established in [16]. Moreover, it was proved in $[16,17]$ that global attractors exist in the class $H_{p e r}^{k}$ for any $k \geq 2$. In this paper, we carry out the bifurcation analysis of the one dimensional problem (1.1) in detail by using the center manifold reduction. 
Let us investigate the eigenvalues of the operator $\mathcal{L}_{\alpha}$ on $H(\tilde{H}$, resp.). By a simple computation, one can find that $\mathcal{L}_{\alpha}$ has an eigenvalue sequence

$$
\beta_{n}(\alpha)=\alpha-\alpha_{n}, \quad \alpha_{n}=\left[1-\left(\frac{n \pi}{\lambda}\right)^{2}\right]^{2}, \quad n=0,1,2, \ldots
$$

with the corresponding eigenvectors

$$
\phi_{0}(x)=\frac{1}{\sqrt{2}}, \quad \phi_{n}(x)=\cos \frac{n \pi x}{\lambda}, \quad \psi_{n}(x)=\sin \frac{n \pi x}{\lambda}
$$

for $n \geq 1$ (in $\tilde{H}, \phi_{0}$ and $\phi_{n}(n \geq 1)$ are only eigenvectors). For our convenience, we denote $\psi_{0}(x)=0$. We note that the eigenvectors are orthogonal to each other and

$$
\left\|\phi_{n}\right\|_{H}=\left\|\psi_{m}\right\|_{H}=\sqrt{\lambda} \quad\left(\left\|\phi_{n}\right\|_{\tilde{H}}=\sqrt{\lambda}, \text { resp. }\right)
$$

for all $n \geq 0$ and $m \geq 1$. Since $\alpha_{n}$ is a quadratic function of $(n \pi / \lambda)^{2}$, each $N \in \mathbb{N}$ has two choices: either

$$
\alpha_{n}>\alpha_{N} \quad \forall n \neq N,
$$

or

$$
\alpha_{n}>\alpha_{N}=\alpha_{N+1} \quad \forall n \neq N, N+1 .
$$

In this paper, we deal with only the first choice. The latter case will be considered in a forthcoming paper.

The main results of this paper are to verify the dynamical bifurcation of the MSHE (1.1) defined in $\tilde{H}$ and $H$. We state the main theorems as follows.

Theorem 1.1. Suppose that (1.4) holds true for some $N \in \mathbb{N} \cup\{0\}$.

(i) If $N=0$, then as $\alpha$ passes through $\alpha_{0}=1$, MSHE (1.1) defined in $\tilde{H}$ bifurcates to two steady points

$$
u= \pm \sqrt{2(\alpha-1)} \phi_{N}+o(\sqrt{\alpha-1}) .
$$

(ii) Suppose that $N>0$ and $\lambda<\sqrt{5 / 2} N \pi$. Then, as $\alpha$ passes through $\alpha_{N}$, MSHE (1.1) defined in $\tilde{H}$ bifurcates to two steady points

$$
u^{ \pm}= \pm \rho_{\alpha} \phi_{N}+o\left(\alpha-\alpha_{N}\right),
$$

where $\rho_{\alpha}=\rho_{\alpha}(N, \mu)>0$ and

$$
\rho_{\alpha}^{2}=\frac{12\left(2 \lambda^{2}-5 N^{2} \pi^{2}\right) \beta_{N}}{9\left(2 \lambda^{2}-5 N^{2} \pi^{2}\right)-4 \mu^{2} N^{2} \pi^{2}}+o\left(\left|\alpha-\alpha_{N}\right|\right) .
$$

Theorem 1.2. Suppose that (1.4) holds true for some $N \in \mathbb{N} \cup\{0\}$.

(i) If $N=0$, then as $\alpha$ passes through $\alpha_{0}=1, M S H E$ (1.1) defined in $H$ bifurcates to two steady points

$$
u= \pm \sqrt{2(\alpha-1)} \phi_{N}+o(\sqrt{\alpha-1}) .
$$


(ii) Suppose that $N>0$ and $\lambda<\sqrt{5 / 2} N \pi$. Then, as $\alpha$ passes through $\alpha_{N}, M S H E$ (1.1) defined in $H$ bifurcates to an attractor $\mathcal{A}_{N}(\alpha)$ which is homeomorphic to $S^{1}$ and consists of the steady solution given by

$$
\left\{u=w_{1} \phi_{N}+w_{2} \psi_{N}+o\left(\alpha-\alpha_{N}\right): w_{1}^{2}+w_{2}^{2}=\rho_{\alpha}^{2}\right\}
$$

The constraint $\lambda<\sqrt{5 / 2} N \pi$ in Theorems 1.1 and 1.2 is natural. As we shall see in the proof of both theorems, this comes from the inequality $\alpha_{2 N}>\alpha_{N}$. This is always true under the condition (1.4). We refer to the attractor $\mathcal{A}_{N}(\alpha)$ in Theorem 1.2 as a $S^{1}$-attractor because it is homeomorphic to $S^{1}$.

It is quite interesting to compare our results with the dynamical bifurcation of two other similar types of phase transition equations: the Swift-Hohenberg equation (SHE):

$$
u_{t}=\alpha u-\left(1+\partial_{x x}\right)^{2} u-u^{3}
$$

and the generalized Swift-Hohenberg equation (GSHE):

$$
u_{t}=\alpha u-\left(1+\partial_{x x}\right)^{2} u+\mu u^{2}-u^{3} .
$$

The MSHE, the SHE, and the GSHE share the same linear part and the only difference of dynamics arises from the nonlinear effect. As in the case of the MSHE, it is known from $[4,8,11,20]$ that under the condition (1.4) and the periodic condition, the SHE and the GSHE bifurcate from the trivial states to $S^{1}$-attractors $\tilde{\mathcal{A}}_{N}(\alpha)$ and $\hat{\mathcal{A}}_{N}(\alpha)$, respectively. However, there are big differences in the structures of $\mathcal{A}_{N}(\alpha), \tilde{\mathcal{A}}_{N}(\alpha)$, and $\hat{\mathcal{A}}_{N}(\alpha)$. First, $\tilde{\mathcal{A}}_{N}(\alpha)$ consists of four static solutions and their connecting orbits. Two of the static solutions are stable points and the others are saddle points. On the other hand, $\hat{\mathcal{A}}_{N}(\alpha)$ consists of static solutions and the direction of the bifurcation depends on the value of $\mu$. Indeed, there is a number $H(N, \lambda, \mu)$ such that if $H(N, \lambda, \mu)>0$, then the bifurcation is subcritical, i.e., the GSHE bifurcates as $\alpha$ passes through $\alpha_{N}$ to the right. If $H(N, \lambda, \mu)<0$, then the bifurcation is supercritical, i.e., the GSHE bifurcates as $\alpha$ passes through $\alpha_{N}$ to the left. In our results for the MSHE, the bifurcated attractor $\mathcal{A}_{N}(\alpha)$ consists of static solutions but there is no dependence on $\mu$ for the direction of the bifurcation.

We prove the above theorems in the next two sections. The main ingredient of proof is the center manifold reduction. If $\alpha$ stays near the critical bifurcation number $\alpha_{N}$, the long time dynamics of the solutions are completely determined from the center manifold about the corresponding eigenspace. Hence, the reduction of MSHE on the center manifold is the key process for the study on bifurcation. In general, it is very difficult to calculate the center manifold function. Recently, Ma and Wang derived a rather simple formula to compute it [13]. We will use this formula to derive the reduced equations on the center manifold. 


\section{Proof of Theorem 1.1}

In this section, we prove Theorem 1.1. We assume that $\alpha$ is slightly bigger than $\alpha_{N}$, namely,

$$
\beta_{N}(\alpha)=o\left(\alpha-\alpha_{N}\right) .
$$

Let $\tilde{E}_{1}=\operatorname{span}\left\{\phi_{N}\right\}$ and $\tilde{E}_{2}=\tilde{E}_{1}^{\perp}$ in $\tilde{H}$. Let $\tilde{P}_{j}: \tilde{H} \rightarrow \tilde{E}_{j}$ be the canonical projection and $\tilde{\mathcal{L}_{j}^{\alpha}}=\left.\mathcal{L}_{\alpha}\right|_{\tilde{E}_{j}}$ for $j=1,2$. For $u \in \tilde{H}$, we write $u=\sum_{n=0}^{\infty} y_{n} \phi_{n}$. If $\tilde{\Phi}: \tilde{E}_{1} \rightarrow \tilde{E}_{2}$ is a center manifold function and $v=\tilde{P}_{1} u=y_{N} \phi_{N}$, then the reduced equation of (1.1) on the center manifold is

$$
\frac{d v}{d t}=\tilde{\mathcal{L}}_{1}^{\alpha} v+\tilde{P}_{1} G\left(y_{N} \phi_{N}+\tilde{\Phi}\left(y_{N} \phi_{N}\right)\right) .
$$

By taking the inner product of (2.7) with $\phi_{N}$, we have the following:

$$
\frac{d y_{N}}{d t}=\beta_{N} y_{N}+g\left(y_{N}\right)
$$

where

$$
g\left(y_{N}\right)=\frac{1}{\lambda}\left\langle G_{2}\left(y_{N} \phi_{N}+\tilde{\Phi}\left(y_{N} \phi_{N}\right)\right), \phi_{N}\right\rangle+\frac{1}{\lambda}\left\langle G_{3}\left(y_{N} \phi_{N}+\tilde{\Phi}\left(y_{N} \phi_{N}\right)\right), \phi_{N}\right\rangle .
$$

On the other hand, from Theorem 3.8 in [13], the center manifold function $\tilde{\Phi}$ can be expressed as

$$
\begin{aligned}
\tilde{\Phi}\left(y_{N} \phi_{N}\right) & =\left(-\tilde{\mathcal{L}_{2}^{\alpha}}\right)^{-1} \tilde{P}_{2} G_{2}\left(y_{N} \phi_{N}\right)+O\left(\left|\beta_{N}\right| \cdot \lambda\left|y_{N}\right|^{2}\right)+o\left(\lambda\left|y_{N}\right|^{2}\right) \\
& =\left(-\tilde{\mathcal{L}_{2}^{\alpha}}\right)^{-1} \tilde{P}_{2} G_{2}\left(y_{N} \phi_{N}\right)+o\left(\left|y_{N}\right|^{2}\right),
\end{aligned}
$$

where the last equality comes from (2.6). We divide the proof into two cases.

\subsection{The case: $N=0$}

Since $G_{2}\left(y_{0} \phi_{0}\right)=0$, it follows from (2.9) that $\tilde{\Phi}\left(y_{0} \phi_{0}\right)=o\left(\left|y_{0}\right|^{2}\right)$. Then

$$
\begin{aligned}
\left\langle G_{2}\left(y_{0} \phi_{0}+\tilde{\Phi}\left(y_{0} \phi_{0}\right)\right), \phi_{0}\right\rangle & =o\left(\left|y_{0}\right|^{3}\right), \\
\left\langle G_{3}\left(y_{0} \phi_{0}+\tilde{\Phi}\left(y_{0} \phi_{0}\right)\right), \phi_{0}\right\rangle & =-\int_{-\lambda}^{\lambda}\left[y_{0} \phi_{0}+\tilde{\Phi}\left(y_{0} \phi_{0}\right)\right]^{3} \cdot \phi_{0} d x \\
& =-\frac{\lambda}{2} y_{0}^{3}+o\left(\left|y_{0}\right|^{3}\right) .
\end{aligned}
$$

Therefore, (2.8) becomes

$$
\frac{d y_{0}}{d t}=\beta_{0} y_{0}-\frac{1}{2} y_{0}^{3}+o\left(\left|y_{0}\right|^{3}\right) .
$$

Hence, we obtain a pitchfork bifurcation as $\alpha$ passes through $\alpha_{0}=1$, which gives two steady state solutions $y_{0}= \pm \sqrt{2(\alpha-1)}+o(\sqrt{\alpha-1})$. 


\subsection{The case: $N \geq 1$}

By direct computation, we have

$$
\begin{aligned}
G_{2}\left(y_{N} \phi_{N}\right) & =\mu\left(y_{N} \phi_{N}\right)_{x}^{2} \\
& =\mu\left(-\frac{N \pi}{\lambda} y_{N} \sin \frac{N \pi x}{\lambda}\right)^{2} \\
& =\mu\left(\frac{N \pi}{\lambda}\right)^{2} \frac{y_{N}^{2}}{2}\left(\sqrt{2} \phi_{0}-\phi_{2 N}\right) .
\end{aligned}
$$

Hence, by (2.9) the center manifold function becomes

$$
\begin{aligned}
\tilde{\Phi}\left(y_{N} \phi_{N}\right) & =\frac{\mu}{2}\left(\frac{N \pi}{\lambda}\right)^{2} y_{N}^{2}\left(-\mathcal{L}_{2}^{\alpha}\right)^{-1}\left(\sqrt{2} \phi_{0}-\phi_{2 N}\right)+o\left(\left|y_{N}\right|^{2}\right) \\
& =\frac{\mu}{2}\left(\frac{N \pi}{\lambda}\right)^{2} y_{N}^{2}\left(-\frac{\sqrt{2} \phi_{0}}{\beta_{0}}+\frac{\phi_{2 N}}{\beta_{2 N}}\right)+o\left(\left|y_{N}\right|^{2}\right) .
\end{aligned}
$$

Here, we used the fact that $\mathcal{L}_{\alpha} \phi_{n}=\beta_{n} \phi_{n}$. Then

$$
\begin{aligned}
& G_{2}\left(y_{N} \phi_{N}+\tilde{\Phi}\left(y_{N} \phi_{N}\right)\right) \\
= & \mu\left\{y_{N} \phi_{N}+\frac{\mu}{2}\left(\frac{N \pi}{\lambda}\right)^{2} y_{N}^{2}\left(-\frac{\sqrt{2} \phi_{0}}{\beta_{0}}+\frac{\phi_{2 N}}{\beta_{2 N}}\right)+o\left(\left|y_{N}\right|^{2}\right)\right\}_{x}^{2} \\
= & \mu\left\{-y_{N}\left(\frac{N \pi}{\lambda}\right) \sin \frac{N \pi x}{\lambda}-y_{N}^{2} \frac{\mu}{2 \beta_{2 N}}\left(\frac{N \pi}{\lambda}\right)^{2}\left(\frac{2 N \pi}{\lambda}\right) \sin \frac{2 N \pi x}{\lambda}+o\left(\left|y_{N}\right|^{2}\right)\right\}^{2} \\
= & y_{N}^{2} \frac{\mu N^{2} \pi^{2}}{\lambda^{2}} \sin ^{2} \frac{N \pi x}{\lambda}+y_{N}^{3} \frac{2 \mu^{2} N^{4} \pi^{4}}{\lambda^{4} \beta_{2 N}} \sin \frac{N \pi x}{\lambda} \sin \frac{2 N \pi x}{\lambda}+o\left(\left|y_{N}\right|^{3}\right) \\
= & y_{N}^{2} \frac{\mu N^{2} \pi^{2}}{\lambda^{2}} \frac{\sqrt{2} \phi_{0}-\phi_{2 N}}{2}+y_{N}^{3} \frac{\mu^{2} N^{4} \pi^{4}}{\lambda^{4} \beta_{2 N}}\left(\phi_{N}-\phi_{3 N}\right)+o\left(\left|y_{N}\right|^{3}\right) .
\end{aligned}
$$

As a consequence,

$$
\begin{aligned}
\frac{1}{\lambda}\left\langle G_{2}\left(y_{N} \phi_{N}+\tilde{\Phi}\left(y_{N} \phi_{N}\right)\right), \phi_{N}\right\rangle & =\frac{1}{\lambda} \int_{-\lambda}^{\lambda} G_{2}\left(y_{N} \phi_{N}+\tilde{\Phi}\left(y_{N} \phi_{N}\right)\right) \cdot \phi_{N} d x \\
& =\frac{\mu^{2} N^{4} \pi^{4}}{\lambda^{4} \beta_{2 N}} y_{N}^{3}+o\left(\left|y_{N}\right|^{3}\right) .
\end{aligned}
$$

On the other hand,

$$
\begin{aligned}
& G_{3}\left(y_{N} \phi_{N}+\tilde{\Phi}\left(y_{N} \phi_{N}\right)\right) \\
= & -\left\{y_{N} \phi_{N}+\frac{\mu}{2}\left(\frac{N \pi}{\lambda}\right)^{2} y_{N}^{2}\left(-\frac{\sqrt{2} \phi_{0}}{\beta_{0}}+\frac{\phi_{2 N}}{\beta_{2 N}}\right)+o\left(\left|y_{N}\right|^{2}\right)\right\}^{3} \\
= & -y_{N}^{3} \cos ^{3} \frac{N \pi x}{\lambda}+o\left(\left|y_{N}\right|^{3}\right) \\
= & -y_{N}^{3} \frac{3 \phi_{N}+\phi_{3 N}}{4}+o\left(\left|y_{N}\right|^{3}\right),
\end{aligned}
$$


DYNAMICAL BIFURCATION OF MODIFIED SWIFT-HOHENBERG EQUATION 1247 which yields that

$$
\begin{aligned}
\frac{1}{\lambda}\left\langle G_{3}\left(y_{N} \phi_{N}+\tilde{\Phi}\left(y_{N}\right)\right), \phi_{N}\right\rangle & =\frac{1}{\lambda} \int_{-\lambda}^{\lambda} G_{3}\left(y_{N} \phi_{N}+\tilde{\Phi}\left(y_{N} \phi_{N}\right)\right) \cdot \phi_{N} d x \\
& =-\frac{3}{4} y_{N}^{3}+o\left(\left|y_{N}\right|^{3}\right) .
\end{aligned}
$$

In the sequel, (2.8) becomes

$$
\frac{d y_{N}}{d t}=\beta_{N} y_{N}-d_{N} y_{N}^{3}+o\left(\left|y_{N}\right|^{3}\right),
$$

where

$$
d_{N}=d_{N}(\alpha, \lambda, \mu)=\frac{3}{4}-\frac{\mu^{2} N^{4} \pi^{4}}{\lambda^{4} \beta_{2 N}} .
$$

We note that (2.11) has two steady points $y_{N}= \pm \rho_{\alpha}$ with $\rho_{\alpha}>0$, where

$$
\rho_{\alpha}^{2}=\frac{\beta_{N}}{d_{N}}=\frac{4 \lambda^{4} \beta_{2 N} \beta_{N}}{3 \lambda^{4} \beta_{2 N}-4 \mu^{2} N^{4} \pi^{4}} .
$$

It follows from (1.4) and (2.6) that

$$
\beta_{2 N}=\alpha_{N}-\alpha_{2 N}+\alpha-\alpha_{N}<0
$$

and hence $\rho_{\alpha}$ is well-defined. Moreover, since

$$
\begin{aligned}
\alpha_{N}-\alpha_{2 N} & =\left(1-\left(\frac{N \pi}{\lambda}\right)^{2}\right)^{2}-\left(1-\left(\frac{2 N \pi}{\lambda}\right)^{2}\right)^{2} \\
& =\frac{3 N^{2} \pi^{2}}{\lambda^{4}}\left(2 \lambda^{2}-5 N^{2} \pi^{2}\right),
\end{aligned}
$$

we have a constraint for $\lambda$ :

$$
\lambda<\sqrt{\frac{5}{2}} N \pi .
$$

The formulas (2.14) and (2.15) also provide an exact form of (2.13) as

$$
\begin{aligned}
\rho_{\alpha}^{2}= & \frac{12\left(2 \lambda^{2}-5 N^{2} \pi^{2}\right) \beta_{N}}{9\left(2 \lambda^{2}-5 N^{2} \pi^{2}\right)-4 \mu^{2} N^{2} \pi^{2}}-\frac{16 \mu^{2} \lambda^{4} \beta_{N}\left(\alpha-\alpha_{N}\right)}{\left(9\left(2 \lambda^{2}-5 N^{2} \pi^{2}\right)-4 \mu^{2} N^{2} \pi^{2}\right)^{2}} \\
& +o\left(\left|\alpha-\alpha_{N}\right|\right) \\
= & \frac{12\left(2 \lambda^{2}-5 N^{2} \pi^{2}\right) \beta_{N}}{9\left(2 \lambda^{2}-5 N^{2} \pi^{2}\right)-4 \mu^{2} N^{2} \pi^{2}}+o\left(\left|\alpha-\alpha_{N}\right|\right) .
\end{aligned}
$$

This completes the proof. 


\section{Proof of Theorem 1.2}

In this section, we prove Theorem 1.2. The case $N=0$ is similar to that of Theorem 1.1 and we omit the detail. Now let us assume that $N \in \mathbb{N}$. As in the proof of Theorem 1.1, the main ingredient is the center manifold reduction.

Let $E_{1}=\operatorname{span}\left\{\phi_{N}, \psi_{N}\right\}$ for $N \geq 1$ and $E_{2}=E_{1}^{\perp}$ in $H$. Let $P_{j}: H \rightarrow E_{j}$ be the canonical projection and $\mathcal{L}_{j}^{\alpha}=\left.\mathcal{L}_{\alpha}\right|_{E_{j}}$ for $j=1,2$. For $u \in H$, we write

$$
u=\sum_{n=0}^{\infty}\left(y_{n} \phi_{n}+z_{n} \psi_{n}\right) .
$$

If $\Phi: E_{1} \rightarrow E_{2}$ is a center manifold function and $v=P_{1} u=y_{N} \phi_{N}+z_{N} \psi_{N}$, then the reduced equation of (1.1) on the center manifold is

$$
\frac{d v}{d t}=\mathcal{L}_{1}^{\alpha} v+P_{1} G\left(y_{N} \phi_{N}+z_{N} \psi_{N}+\Phi\left(y_{N} \phi_{N}+z_{N} \psi_{N}\right)\right) .
$$

By taking the inner product of (3.16) with $\phi_{N}$ and $\psi_{N}$, we have the following:

$$
\left\{\begin{array}{l}
\frac{d y_{N}}{d t}=\beta_{N} y_{N}+F_{1}\left(y_{N}, z_{N}\right), \\
\frac{d z_{N}}{d t}=\beta_{N} z_{N}+F_{2}\left(y_{N}, z_{N}\right) .
\end{array}\right.
$$

Here,

$$
\begin{aligned}
F_{1}\left(y_{N}, z_{N}\right)= & \frac{1}{\lambda}\left\langle G_{2}\left(y_{N} \phi_{N}+z_{N} \psi_{N}+\Phi\left(y_{N} \phi_{N}+z_{N} \psi_{N}\right)\right), \phi_{N}\right\rangle \\
& +\frac{1}{\lambda}\left\langle G_{3}\left(y_{N} \phi_{N}+z_{N} \psi_{N}+\Phi\left(y_{N} \phi_{N}+z_{N} \psi_{N}\right)\right), \phi_{N}\right\rangle
\end{aligned}
$$

and

$$
\begin{aligned}
F_{2}\left(y_{N}, z_{N}\right)= & \frac{1}{\lambda}\left\langle G_{2}\left(y_{N} \phi_{N}+z_{N} \psi_{N}+\Phi\left(y_{N} \phi_{N}+z_{N} \psi_{N}\right)\right), \psi_{N}\right\rangle \\
& +\frac{1}{\lambda}\left\langle G_{3}\left(y_{N} \phi_{N}+z_{N} \psi_{N}+\Phi\left(y_{N} \phi_{N}+z_{N} \psi_{N}\right)\right), \psi_{N}\right\rangle .
\end{aligned}
$$

In the following, we compute $F_{1}$ and $F_{2}$.

First, we observe from Theorem 3.8 in [13], the center manifold function $\Phi$ can be expressed as

$$
\begin{aligned}
\Phi\left(y_{N} \phi_{N}+z_{N} \psi_{N}\right)= & \left(-\mathcal{L}_{2}^{\alpha}\right)^{-1} P_{2} G_{2}\left(y_{N} \phi_{N}+z_{N} \psi_{N}\right) \\
& +O\left(\left|\beta_{N}\right| \cdot \lambda\left(y_{N}^{2}+z_{N}^{2}\right)\right)+o\left(\lambda\left(y_{N}^{2}+z_{N}^{2}\right)\right) \\
= & \left(-\mathcal{L}_{2}^{\alpha}\right)^{-1} P_{2} G_{2}\left(y_{N} \phi_{N}+z_{N} \psi_{N}\right)+o\left(\left(y_{N}^{2}+z_{N}^{2}\right)\right),
\end{aligned}
$$

where the last equality comes from (2.6). By direct computation, we have

$$
\begin{aligned}
& G_{2}\left(y_{N} \phi_{N}+z_{N} \psi_{N}\right) \\
= & \mu\left(y_{N} \phi_{N}+z_{N} \psi_{N}\right)_{x}^{2} \\
= & \mu\left(-\frac{N \pi}{\lambda} y_{N} \sin \frac{N \pi x}{\lambda}+\frac{N \pi}{\lambda} z_{N} \cos \frac{N \pi x}{\lambda}\right)^{2}
\end{aligned}
$$


DYNAMICAL BIFURCATION OF MODIFIED SWIFT-HOHENBERG EQUATION 1249

$$
\begin{aligned}
& =\mu\left(\frac{N \pi}{\lambda}\right)^{2}\left[y_{N}^{2} \frac{\sqrt{2} \phi_{0}-\phi_{2 N}}{2}-y_{N} z_{N} \psi_{2 N}+z_{N}^{2} \frac{\sqrt{2} \phi_{0}+\phi_{2 N}}{2}\right] \\
& =\mu\left(\frac{N \pi}{\lambda}\right)^{2}\left[\frac{\sqrt{2}\left(y_{N}^{2}+z_{N}^{2}\right)}{2} \phi_{0}-\frac{y_{N}^{2}-z_{N}^{2}}{2} \phi_{2 N}-y_{N} z_{N} \psi_{2 N}\right] .
\end{aligned}
$$

Hence, by (3.18) the center manifold function becomes

$$
\begin{aligned}
& \Phi\left(y_{N} \phi_{N}+z_{N} \psi_{N}\right) \\
= & -\mu\left(\frac{N \pi}{\lambda}\right)^{2}\left[\frac{\sqrt{2}\left(y_{N}^{2}+z_{N}^{2}\right)}{2} \frac{\phi_{0}}{\beta_{0}}-\frac{y_{N}^{2}-z_{N}^{2}}{2} \frac{\phi_{2 N}}{\beta_{2 N}}-y_{N} z_{N} \frac{\psi_{2 N}}{\beta_{2 N}}\right]+o\left(y_{N}^{2}+z_{N}^{2}\right) .
\end{aligned}
$$

So, we obtain that

$$
\begin{aligned}
& G_{2}\left(y_{N} \phi_{N}+z_{N} \psi_{N}+\Phi\left(y_{N} \phi_{N}+z_{N} \psi_{N}\right)\right) \\
= & \mu\left(y_{N} \phi_{N}+z_{N} \psi_{N}+\Phi\left(y_{N} \phi_{N}+z_{N} \psi_{N}\right)\right)_{x}^{2} \\
=\mu\{ & -y_{N} \frac{N \pi}{\lambda} \psi_{N}+z_{N} \frac{N \pi}{\lambda} \phi_{N}-2 \mu\left(\frac{N \pi}{\lambda}\right)^{3}\left[\frac{y_{N}^{2}-z_{N}^{2}}{2 \beta_{2 N}} \psi_{2 N}-\frac{y_{N} z_{N}}{\beta_{2 N}} \phi_{2 N}\right] \\
& \left.\quad+o\left(y_{N}^{2}+z_{N}^{2}\right)\right\}^{2} \\
=\mu\left\{y_{N}^{2}\left(\frac{N \pi}{\lambda}\right)^{2} \psi_{N}^{2}+z_{N}^{2}\left(\frac{N \pi}{\lambda}\right)^{2} \phi_{N}^{2}-2 y_{N} z_{N}\left(\frac{N \pi}{\lambda}\right)^{2} \phi_{N} \psi_{N}\right. & \quad+\frac{2 \mu}{\beta_{2 N}}\left(\frac{N \pi}{\lambda}\right)^{4}\left[\left(y_{N}^{3}-y_{N} z_{N}^{2}\right) \psi_{N} \psi_{2 N}-2 y_{N}^{2} z_{N} \psi_{N} \phi_{2 N}\right. \\
& \left.\left.\quad-\left(y_{N}^{2} z_{N}-z_{N}^{3}\right) \phi_{N} \psi_{2 N}+2 y_{N} z_{N}^{2} \phi_{N} \phi_{2 N}\right]+o\left(\left|y_{N}\right|^{3}+\left|z_{N}\right|^{3}\right)\right\} .
\end{aligned}
$$

By elementary properties of the trigonometric functions, we obtain

$$
\begin{aligned}
& G_{2}\left(y_{N} \phi_{N}+z_{N} \psi_{N}+\Phi\left(y_{N} \phi_{N}+z_{N} \psi_{N}\right)\right) \\
= & \frac{\mu N^{2} \pi^{2}}{\lambda^{2}}\left[y_{N}^{2} \frac{\sqrt{2} \phi_{0}-\phi_{2 N}}{2}+z_{N}^{2} \frac{\sqrt{2} \phi_{0}-\phi_{2 N}}{2}-y_{N} z_{N} \psi_{2 N}\right] \\
& +\frac{2 \mu^{2} N^{4} \pi^{4}}{\lambda^{4} \beta_{2 N}}\left[\left(y_{N}^{3}-y_{N} z_{N}^{2}\right) \frac{\phi_{N}-\phi_{3 N}}{2}-y_{N}^{2} z_{N}\left(-\psi_{N}+\psi_{3 N}\right)\right. \\
& \left.\quad-\left(y_{N}^{2} z_{N}-z_{N}^{3}\right) \frac{\psi_{N}+\psi_{3 N}}{2}+y_{N} z_{N}^{2}\left(\phi_{N}+\phi_{3 N}\right)\right]+o\left(\left|y_{N}\right|^{3}+\left|z_{N}\right|^{3}\right) \\
= & \frac{\mu N^{2} \pi^{2}}{\lambda^{2}}\left[\frac{y_{N}^{2}+z_{N}^{2}}{\sqrt{2}} \phi_{0}-\frac{y_{N}^{2}+z_{N}^{2}}{2} \phi_{2 N}-y_{N} z_{N} \psi_{2 N}\right] \\
& +\frac{\mu^{2} N^{4} \pi^{4}}{\lambda^{4} \beta_{2 N}}\left[\left(y_{N}^{3}+y_{N} z_{N}^{2}\right) \phi_{N}-\left(y_{N}^{3}-3 y_{N} z_{N}^{2}\right) \phi_{3 N}\right. \\
& \left.+\left(y_{N}^{2} z_{N}+z_{N}^{3}\right) \psi_{N}-\left(3 y_{N}^{2} z_{N}-z_{N}^{3}\right) \psi_{3 N}\right]+o\left(\left|y_{N}\right|^{3}+\left|z_{N}\right|^{3}\right) .
\end{aligned}
$$

As a consequence, we are led to

$$
\frac{1}{\lambda}\left\langle G_{2}\left(y_{N} \phi_{N}+z_{N} \psi_{N}+\Phi\left(y_{N} \phi_{N}+z_{N} \psi_{N}\right)\right), \phi_{N}\right\rangle
$$




$$
\begin{aligned}
= & \frac{\mu^{2} N^{4} \pi^{4}}{\lambda^{4} \beta_{2 N}}\left(y_{N}^{3}+y_{N} z_{N}^{2}\right)+o\left(\left|y_{N}\right|^{3}+\left|z_{N}\right|^{3}\right), \\
& \frac{1}{\lambda}\left\langle G_{2}\left(y_{N} \phi_{N}+z_{N} \psi_{N}+\Phi\left(y_{N} \phi_{N}+z_{N} \psi_{N}\right)\right), \psi_{N}\right\rangle \\
= & \frac{\mu^{2} N^{4} \pi^{4}}{\lambda^{4} \beta_{2 N}}\left(y_{N}^{2} z_{N}+z_{N}^{3}\right)+o\left(\left|y_{N}\right|^{3}+\left|z_{N}\right|^{3}\right) .
\end{aligned}
$$

On the other hand,

$$
\begin{aligned}
& G_{3}\left(y_{N} \phi_{N}+z_{N} \psi_{N}+\Phi\left(y_{N} \phi_{N}+z_{N} \psi_{N}\right)\right) \\
= & -\left(y_{N} \phi_{N}+z_{N} \psi_{N}+\Phi\left(y_{N} \phi_{N}+z_{N} \psi_{N}\right)\right)^{3} \\
= & -y_{N}^{3} \phi_{N}^{3}-3 y_{N}^{2} z_{N} \phi_{N}^{2} \psi_{N}-3 y_{N} z_{N}^{2} \phi_{N} \psi_{N}^{2}-z_{N}^{3} \psi_{N}^{3}+o\left(\left|y_{N}\right|^{3}+\left|z_{N}\right|^{3}\right) \\
= & -y_{N}^{3} \frac{3 \phi_{N}+\phi_{3 N}}{4}-3 y_{N}^{2} z_{N} \frac{\psi_{N}+\psi_{3 N}}{4}-3 y_{N} z_{N}^{2} \frac{\phi_{N}-\phi_{3 N}}{4}-z_{N}^{3} \frac{3 \psi_{N}-\psi_{3 N}}{4} \\
& +o\left(\left|y_{N}\right|^{3}+\left|z_{N}\right|^{3}\right) \\
= & -\frac{1}{4}\left[3\left(y_{N}^{3}+y_{N} z_{N}^{2}\right) \phi_{N}+\left(y_{N}^{3}-3 y_{N} z_{N}^{2}\right) \phi_{3 N}+3\left(y_{N}^{2} z_{N}+z_{N}^{3}\right) \psi_{N}\right. \\
& \left.\quad+\left(3 y_{N}^{2} z_{N}-z_{N}^{3}\right) \psi_{3 N}\right]+o\left(\left|y_{N}\right|^{3}+\left|z_{N}\right|^{3}\right),
\end{aligned}
$$

which yields that

$$
\begin{aligned}
& \frac{1}{\lambda}\left\langle G_{3}\left(y_{N} \phi_{N}+z_{N} \psi_{N}+\Phi\left(y_{N} \phi_{N}+z_{N} \psi_{N}\right)\right), \phi_{N}\right\rangle \\
= & -\frac{3}{4}\left(y_{N}^{3}+y_{N} z_{N}^{2}\right)+o\left(\left|y_{N}\right|^{3}+\left|z_{N}\right|^{3}\right), \\
& \frac{1}{\lambda}\left\langle G_{3}\left(y_{N} \phi_{N}+z_{N} \psi_{N}+\Phi\left(y_{N} \phi_{N}+z_{N} \psi_{N}\right)\right), \psi_{N}\right\rangle \\
= & -\frac{3}{4}\left(y_{N}^{2} z_{N}+z_{N}^{3}\right)+o\left(\left|y_{N}\right|^{3}+\left|z_{N}\right|^{3}\right) .
\end{aligned}
$$

In the sequel, (3.17) becomes

$$
\frac{d \mathbf{y}}{d t}=\beta_{N} \mathbf{y}-\mathbf{F}(\mathbf{y})+o\left(|\mathbf{y}|^{3}\right),
$$

where $\mathbf{y}=\left(y_{N}, z_{N}\right)$ and

$$
\mathbf{F}(\mathbf{y})=d_{N}\left(y_{N}^{3}+y_{N} z_{N}^{2}, y_{N}^{2} z_{N}+z_{N}^{3}\right) .
$$

Here, $d_{N}$ is the number defined by $(2.12)$. Since $\beta_{2 N}<0$, we obtain that $d_{N}>0$. Furthermore, since

$$
\langle\mathbf{F}(\mathbf{y}), \mathbf{y}\rangle=d_{N}\left(y_{N}^{2}+z_{N}^{2}\right)^{2}=d_{N}|\mathbf{y}|^{4},
$$

we have the following:

$$
d_{N}|\mathbf{y}|^{4} \leq\langle\mathbf{F}(\mathbf{y}), \mathbf{y}\rangle \leq 2 d_{N}|\mathbf{y}|^{4} .
$$

This implies by Theorem 5.10 of [13] that (3.19) bifurcates from the trivial solution to an attractor $\mathcal{A}_{N}(\alpha)$ as $\alpha$ passes through $\alpha_{N}$. Moreover, $\mathcal{A}_{N}(\alpha)$ is homeomorphic to $S^{1}$. 
We recall that the MSHE (1.1) is invariant under the even periodic condition. We have seen that the MSHE bifurcates an attractor in $\tilde{H}$ consisting of two steady solutions $\pm \rho_{\alpha} \phi_{N}+o\left(\alpha-\alpha_{N}\right)$. We also note that the MSHE is invariant in $H$ under the spatial translation. As a consequence, the static solution $u=$ $\rho_{\alpha} \phi_{N}+o\left(\alpha-\alpha_{N}\right)$ generates one parameter family of static solutions as follows: for $\theta \in \mathbb{R}$,

$$
\begin{aligned}
& \rho_{\alpha} \cos \left(\frac{N \pi}{\lambda}(x+\theta)\right)+o\left(\alpha-\alpha_{N}\right) \\
= & \rho_{\alpha} \cos \frac{N \pi \theta}{\lambda} \cdot \cos \frac{N \pi x}{\lambda}+\rho_{\alpha} \sin \frac{N \pi \theta}{\lambda} \cdot \cos \frac{N \pi x}{\lambda}+o\left(\alpha-\alpha_{N}\right) \\
= & w_{1} \phi_{N}+w_{2} \psi_{N}+o\left(\alpha-\alpha_{N}\right) .
\end{aligned}
$$

Since $w_{1}^{2}+w_{2}^{2}=\rho_{\alpha}^{2}$, this set of static solutions form an invariant circle. It is obvious that this circle is contained in the attractor $\mathcal{A}_{N}(\alpha)$. Since $\mathcal{A}_{N}(\alpha)$ is already homeomorphic to $S^{1}$, we may conclude that $\mathcal{A}_{N}(\alpha)$ consists of static solutions. This finished the proof.

\section{References}

[1] M. Bestehorn and H. Haken, Transient patterns of the convection instability: A modelcalculation, Z. Phys. B 57 (1984), 329-333.

[2] Y. Choi and J. Han, Dynamical bifurcation of the damped Kuramoto-Sivashinsky equation, J. Math. Anal. Appl. 421 (2015), 383-398.

[3] Y. Choi, J. Han, and C.-H. Hsia, Bifurcation analysis of the damped KuramotoSivashinsky equation with respect to the period, Discrete Contin. Dyn. Syst. Ser. B 20 (2015), no. 7, 1933-1957.

[4] Y. Choi, J. Han, and J. Park, Dynamical bifurcation of the generalized Swift-Hohenberg equation, Int. J. Bifurcat. Chaos 25 (2015), no. 8.

[5] M. Cross and P. Hohenbrg, Pattern formation outside equilibrium, Rev. Mod. Phys. 65 (1993), 581-1112.

[6] A. Doleman, B. Sandstede, A. Scheel, and G. Schneider, Propagation of hexagonal patterns near onset, Euro. J. Appl. Math. 14 (2003), no. 9, 85-110.

[7] N. Duan and W. Gao, Optimal control of a modified Swift-Hohenberg equation, Electron. J. Differential Equations 2012 (2012), no. 155, 1-12.

[8] H. Gao and Q. Xiao, Bifurcation analysis of the $1 D$ and 2D generalized Swift-Hohenberg equation, Internat. J. Bifur. Chaos Appl. Sci. Engrg. 20 (2010), no. 3, 619-643.

[9] J. P. Gollub and J. S. Langer, Pattern formation in nonequilibrium physics, Rev. Mod. Phys. 71 (1999), 396-403.

[10] J. Han and C.-H. Hsia, Dynamical bifurcation of the two dimensional Swift-Hohenberg equation with odd periodic condition, Discrete Contin. Dyn. Syst. Ser. B 17 (2012), no. 7, 2431-2449.

[11] J. Han and M. Yari, Dynamic bifurcation of the periodic Swift-Hohenberg equation, Bull. Korean Math. Soc. 49 (2012), no. 5, 923-937.

[12] M. Hilali, S. Métens, P. Borckmans, and G. Dewwl, Pattern selection in the generalized Swift-Hohenberg model, Phys. Rev. E 51 (1995), 2046-2052.

[13] T. Ma and S. Wang, Bifurcation Theory and Applications, World Scientific, 2005.

[14] L. A. Peletier and V. Rottschäfer, Pattern selection of solutions of the Swift-Hohenberg equations, Phys. D 194 (2004), no. 1-2, 95-126.

[15] L. Peletier and J. Williams, Some canonical bifurcations in the Swift-Hohenberg equation, SIAM J. Appl. Dyn. Syst. 6 (2007), no. 1, 208-235. 
[16] M. Polat, Global attractor for a modified Swift-Hohenberg equation, Comput. Math. Appl. 57 (2009), no. 1, 62-66.

[17] L. Song, Y. Zhang, and T. Ma, Global attractor for a modified Swift-Hohenberg equation in $H^{k}$ spaces, Nonlinear Anal. 72 (2010), 183-191.

[18] J. Swift and P. Hohenberg, Hydrodynamic fluctuations at the convective instability, Phys. Rev. A 15 (1977), 319

[19] Q. Xiao and H. Gao, Bifurcation analysis of the Swift-Hohenberg equation with quintic nonlinearity, Internat. J. Bifur. Chaos Appl. Sci. Engrg. 19 (2009), no. 9, 2927-2937.

[20] M. Yari, Attractor bifurcation and final patterns of the $N$-dimensional and generalized Swift-Hohenberg equations, Discrete Contin. Dyn. Syst. Ser. B 7 (2007), no. 2, 441-456.

[21] X. Zhao, B. Liu, P. Zhang, W. Zhang, and F. Liu, Fourier spectral method for the modified Swift-Hohenberg equation, Adv. Difference Eqns. 2013 (2013), no. 156, 1-19.

Division of General Education

KWANGWOON UNIVERSITY

SEOul 139-701, Korea

E-mail address: yuncherl@kw.ac.kr 